\title{
Raising Children in the Context of Rapid Social Change: Parenting Self-Efficacy of South Korean Mothers
}

\author{
Kaley N. Curtis $\mathbb{D}^{1} \cdot$ Ayumi Nagase $^{2} \cdot$ Soojung $\mathrm{Kim}^{3} \cdot$ Susan D. Holloway ${ }^{4}$
}

Accepted: 9 December 2021 / Published online: 5 January 2022

(C) The Author(s) 2022

\begin{abstract}
Given recent sociocultural and economic changes that have created significant pressures on South Korean mothers, we were interested in understanding the factors that might contribute to their sense of competence in taking on the challenges of childrearing. We examined several family conditions theoretically supportive of mothers' parenting self-efficacy (PSE), specifically whether mothers' PSE was associated with their marital satisfaction and with their children's competence (social and academic). We then examined whether PSE was related to life stress, as well as whether life stress moderated the relationship of PSE to the proximal marital and child variables. Participants included 234 South Korean mothers of preprimary-aged children. Mothers completed a survey reporting on PSE, life stress, and marital satisfaction. Teachers provided ratings of children's competence. Regression analyses indicated that mothers who experienced lower life stress reported higher PSE after controlling for sociodemographic characteristics. Neither marital satisfaction nor child competence was associated with PSE. A second set of regressions tested the same set of predictors, in addition to the interactions of life stress with marital satisfaction and of life stress with child competence. Results indicated that for mothers reporting lower life stress, greater marital satisfaction was associated with higher PSE. Unexpectedly, for mothers experiencing higher life stress, lower marital satisfaction was associated with higher PSE. These findings support theoretical formulations linking PSE with marital support and life stress, while suggesting a need to further investigate the association among these factors in a variety of situational contexts, particularly those that are appraised as stressful.
\end{abstract}

Keywords Parenting $\cdot$ Parenting self-efficacy $\cdot$ Marital satisfaction $\cdot$ South Korea $\cdot$ Pre-primary

\section{Highlights}

- Examined two family-level factors associated with PSE of South Korean mothers: marital satisfaction and children's academic and social competence.

- Examined life stress as an environmental factor that may be associated with PSE.

- Explored whether life stress moderated the relationship between PSE and family-level factors.

- Lower stress was related to higher PSE, controlling for family sociodemographic factors.

- Marital satisfaction was positively related to PSE for mothers experiencing relatively low stress, and negatively related for those experiencing high stress.

Kaley N. Curtis

kaley_curtis@berkeley.edu

1 Department of Psychology, University of California Berkeley, 2121 Berkeley Way, Berkeley, CA 94720-1650, USA

2 Department of Early Childhood Studies, Sonoma State University, 1801 East Cotati Avenue, Rohnert Park, CA 94928, USA
3 Department of Child and Youth Welfare, Pyeongtaek University, 111 Yongyi-Dong, Pyeongtaek-si, Gyeonggi-Do 450-701, South Korea

4 Graduate School of Education, University of California Berkeley, 2121 Berkeley Way, Berkeley, CA 94720-1650, USA 
An ecological theory posits that contextual factors at multiple levels of influence can affect the formation of an individual's goals, self-perceptions, and expectations (Bronfenbrenner \& Morris, 2006). In this study, we focus on how two factors at the level of the microsystem (marital satisfaction; child social and academic competence) are related to South Korean mothers' appraisal of their competence in childrearing (parenting self-efficacy [PSE]). We further examine how mothers' life stress is related to their PSE, and whether life stress moderates the association between PSE and the two microsystem variables.

According to Bronfenbrenner, microsystem factors that affect an individual include the support and guidance available from important social groups surrounding the individual, including family, peers, religious institutions, and the like. One key microsystem element, satisfaction with the marital relationship, has been an important predictor of PSE in studies conducted in the United States and Western Europe (Bornstein et al., 2003). In contrast to many of these Western countries, traditional gender roles with respect to family life are more strongly endorsed in South Korea (albeit more so by men than women), and most aspects of household management, including childrearing, are seen as largely the responsibility of mothers, with fathers' role consisting primarily of providing financial support (Cha \& Song, 2017, Chung et al., 2015, Hong \& Lee, 2019, Kwon \& Roy, 2007, Jeong \& Jeon, 2018, Shwalb et al., 2004, Yoo, 2020). Given these national differences in expectations regarding husbands' support, we asked whether mothers' satisfaction with the behavioral and psychological support provided by their husbands is associated with their PSE in a South Korean sample, as it is in studies conducted in other countries.

A second microsystem factor associated with PSE pertains to the personal characteristics of the child. Parenting self-efficacy is much more likely to be high when parents experience a sense of success in their attempts to guide and support their children (Jones \& Prinz, 2005). Children whose social development is on track tend to be more responsive to parental guidance, and hence provide a source of reinforcement to parents that bolster parental self-confidence. Similarly, when their children's academic potential is apparent, parents tend to conclude they are "doing something right". In South Korea, children's academic achievement may be a particularly strong indicator to mothers concerning their progress and success in childrearing because it has traditionally been valued more highly in South Korea than in many Western societies. Indeed, $70 \%$ of South Korean adults aged 25 to 34 have a tertiary degree (OECD Education GPS, 2021). It stands to reason that mothers whose children are doing well in their early academic life are more likely to feel efficacious than those whose children are not progressing as rapidly (Park et al., 2011). In addition to their regular schoolwork, a large majority of South Korean children attend private tutoring academies (hagwon) (Bak, 2017, Koo, 2007, Korean Statistical Information Service, 2015a). South Korean children are closely assessed and evaluated in these supplementary programs as well as in their conventional schools, rendering this information available and salient to mothers. Accordingly, we obtained teacher ratings of children's social and academic competence (hereafter referred to as child competence) and examined its relationship to PSE.

A third question we examined in the current study was whether the degree of life stress that women experience is associated with their PSE. While this is a common finding in studies conducted in Western countries, it has been studied far less frequently with respect to South Korea (Hong \& Lee, 2019). In contemporary South Korean society, many families are experiencing financial stressors. There is a growing gap between the poorest and wealthiest South Korean citizens, with the top 20\% earning more than five times as much as the bottom $20 \%$. In the last five years, a substantial number of middle-class families have experienced economic hardship, and rising property costs have left many of them unable to invest in a home (C. Kim, 2020). Additionally, the supplementary education that most children receive comes at a price; in 2019 , the overall ratio of private education expense to household income was 0.07 , with an even higher proportion in lower- and middle-income families (S. Lee \& Shouse, 2011, "Students' private education", 2020). Gender norms are another factor that can exacerbate the life stress among South Korean mothers. While social changes have made it easier for women to participate in the workforce, their financial compensation relative to their male counterparts is among the lowest of economically comparable nations (World Economic Forum, 2019). Because South Korean women do most of the housework and childrearing, those who are employed may bear a stressful double burden of household management and outside employment for which they are often unfairly compensated (Choi et al., 2018, Jeong \& Jeon, 2018).

In summary, our study examines three key elements of family life in the context of South Korea, each of which is potentially associated with mothers' sense of PSE: marital satisfaction, child competence, and life stress. We expected that each of these factors would be significantly related to PSE. Additionally, we examined whether life stress moderates the relationship between PSE and marital satisfaction as well as the relationship between PSE and child competence. In other words, is the association of PSE to marital satisfaction or to child competence dependent on the amount of life stress that the mothers are experiencing in this particular economic and cultural context? 


\section{Theoretical Perspectives on PSE}

Grounded in social cognitive theory, self-efficacy judgments reflect a cognitive tendency to appraise one's own actions relative to personal goals and expectations (Bandura, 1977, 1997). According to Bandura, individuals form selfefficacy judgments with respect to particular domains of life, such as the academic domain or the parenting domain. Thus, PSE refers to parents' confidence in their ability to engage in the activities they judge to be important to their role as a parent (Jones \& Prinz, 2005). This appraisal is in turn associated with more effective parenting, particularly in the face of challenges (Coleman \& Karraker, 1998, 2000). Many studies conducted in a variety of countries have found that mothers who believe that they have sufficient skill to be a good parent are able to manage their anxiety and maintain the focus and energy needed to engage in positive parenting behaviors (e.g., Chau \& Giallo, 2015, Dumka et al., 2010).

While the impetus toward self-appraisal is posited to be universal, the nature of the appraisal itself is assumed to be a subjective process that is affected by a variety of factors, some of which are personal and others which are societal or cultural in nature (Bandura, 1995). In particular, certain parenting supports, as well as particular child attributes, are valorized within particular cultural settings, highlighting the importance of studying reciprocal relations among cultural factors, parenting supports, and children's competencies (Harkness et al., 2007). Previous work with South Korean families indicated that high PSE is powerfully linked to more positive parenting behaviors, which in turn have been associated with child competence (S. Kim \& Holloway, 2018, Shim \& Lim, 2019).

\section{Children's Social and Academic Competence as an Indicator of Parenting Success}

According to social cognitive theory, one's previous experiences of success and failure with respect to a domain are powerful predictors of self-efficacy with regard to future challenges in that domain. Accordingly, it stands to reason that parents whose children exhibit academic or social difficulties may judge themselves as less competent parents than those whose children are not experiencing such problems (Coleman \& Karraker, 2003, Donovan \& Leavitt, 1989, Gross \& Tucker, 1994, Shim \& Lim, 2019). In South Korea, as we have noted, parents place great importance on children's educational success and higher school achievement can be seen as a marker of good parenting. In our study, we were able to assess this child competence hypothesis by examining whether children's social and academic competence as rated by their teachers was associated with higher maternal PSE.

\section{Marital Satisfaction}

According to social cognitive theory, another powerful predictor of self-efficacy is social support in the form of emotional encouragement or instrumental assistance in completing a task (Bandura, 1977, 1997). In the parenting domain, the support of a spouse or partner is considered a crucial factor in developing and maintaining PSE (Erel \& Burman, 1995, Schneewind, 1995). A supportive and engaged partner can take on childrearing work, provide emotional support, and model potentially successful responses and strategies in the context of parenting challenges. Research conducted in the United States and Europe, while somewhat sparse, generally supports the notion that partner support is associated with the PSE of mothers as well as fathers (Merrifield \& Gamble, 2012, see also Bouchard et al., 2007, Suzuki et al., 2009). It should be noted that in a substantial number of national contexts parents are not married to their partners nor are couples necessarily heterosexual. To date, however, most research conducted with East Asian families reflects the heteronormative model that is predominant in that region. Accordingly, we will henceforth refer primarily to the literature on married heterosexual couples, but acknowledge the limitation of scholarship in this area.

Of course, sociocultural contexts differ with respect to how spouses are expected to address each other's psychological needs (Yeung, 2013). Research on marital satisfaction and PSE in Asian countries is scarce, but one study conducted in Japan suggests that the relatively low PSE expressed by Japanese mothers relative to their American counterparts is partially attributable to their correspondingly low marital satisfaction (Suzuki et al., 2009). On the other hand, Chinese mothers who reported greater marital satisfaction perceived themselves as less stressed but did not differ from less satisfied women in terms of PSE (Kwok et al., 2015). Given the limited empirical evidence on this question, we made a tentative prediction based largely on the theory that marital satisfaction and PSE would be positively related in our sample.

\section{Life Stress and its Association to PSE}

A central focus of the present study is to ascertain whether mothers who report higher levels of life stress are more likely to perceive themselves as less efficacious parents. Few studies have examined whether stressful conditions undermine parents' sense of efficacy in raising their children (Jones \& Prinz, 2005), but there is some evidence that American mothers who feel generally overloaded by work and family responsibilities are also more likely to have lower PSE (Erdwins et al., 2001). These authors emphasize 
the transactional nature of this association; that is, experiences of stress and perceptions of parenting ability are likely to inform each other in a cyclical way.

In addition to examining the direct association of life stress to PSE, we were also interested in examining the role of stress as a moderator. In contrast to the main effects model, which proposes social support to have a direct effect on outcomes irrespective of whether individuals are under high or low levels of stress, the stress-buffering model proposes that social support is differentially related to outcomes as a function of the amount of stress individuals are experiencing (Cohen \& Wills, 1985, Cohen et al., 2000). Specifically, we asked whether the association of PSE to marital satisfaction and to children's competence depends on mothers' degree of life stress. In general, we hypothesized that these proximal sources of affirmation may be associated with PSE only when mothers' stress levels are relatively mild, whereas when stress is high, even these positive proximal factors may not be sufficient to boost PSE.

\section{Current Study}

Our study focused on mothers of children enrolled in preprimary school. In South Korea, primary school begins in first grade, but over $90 \%$ of South Korean children attend either private or public programs prior to that time (National Center for Education Statistics, 2020). In this pivotal period, parents whose children are in a preprimary program have an opportunity to gain insight into their children's status compared to their peers, and to obtain teachers' evaluation of their children's skills. The following research questions were addressed in the current study:

1. Controlling for family sociodemographic characteristics (household income, child age, child gender, maternal age, maternal labor force participation, and maternal education), is there an association between marital satisfaction and PSE?

2. Controlling for these family factors, is child competence related to mothers' PSE?

3. Controlling for these family factors, is mothers' life stress associated with their PSE?

4. Is the relationship of PSE to marital satisfaction and to children's competence moderated by mothers' life stress?

Based on past findings of research conducted in South Korea as well as other national contexts, we hypothesized that higher PSE would be associated with higher marital satisfaction, children exhibiting more competence, and lower life stress. However, there is little research focused on the role of maternal stress in moderating the association
Table 1 Descriptive statistics of study variables

\begin{tabular}{lccccl}
\hline Variable & $N$ & Mean & $S D$ & Minimum & Maximum \\
\hline Household income $^{\mathrm{a}}$ & 212 & 3.91 & 1.20 & 1.00 & 7.00 \\
Child age (months) $^{234}$ & 70.76 & 6.81 & 58.00 & 81.00 \\
Child gender $^{\mathrm{b}}$ & 234 & 0.50 & 0.50 & 0 & 1.00 \\
Mother age (years) & 222 & 37.4 & 4.01 & 27.0 & 48.0 \\
Mother labor force $^{\text {participation }}{ }^{\mathrm{c}}$ & 234 & 13.13 & 20.5 & 0 & 90 \\
Mother education $^{\mathrm{d}}$ & 233 & 0.23 & 0.42 & 0 & 1.00 \\
Mother life stress & 231 & 2.85 & 0.54 & 1.11 & 4.44 \\
Mother marital & 231 & 3.50 & 0.82 & 1.20 & 5.00 \\
satisfaction & & & & & \\
Child competence & 234 & 3.53 & 0.67 & 1.48 & 5.00 \\
Mother PSE & 231 & 3.97 & 0.79 & 1.89 & 5.89
\end{tabular}

${ }^{\mathrm{a}} 1=$ Under KRW 12,000,000; 2 = KRW 12,010,000-24,000,000;

$3=$ KRW 24,010,000-36,000,000; $4=$ KRW 36,010,000-50,000,000;

$5=$ KRW 50,010,000-75,000,000; $6=$ KRW 75,010,000-100,000,000;

7 = Over KRW 100,000,000

${ }^{\mathrm{b}} 0=$ male; $1=$ female

${ }^{\mathrm{c}}$ Measured in hours worked per week

${ }^{\mathrm{d}}$ Coded dichotomously with $0=$ below college degree attainment; $1=$ college degree attainment or above

between PSE and each of these proximal factors (marital satisfaction, child competence). We, therefore, considered this final question to be exploratory in nature, although we suspected that features of the family context would be more strongly associated with PSE when mothers were experiencing relatively low levels of life stress and could attend more carefully to specific proximal family conditions.

\section{Method}

\section{Participants}

The participants were 234 mothers of preprimary-aged children living in the Incheon metropolitan area in South Korea. As Table 1 indicates, mothers' average age was 37.41 years $(S D=4.01)$ and the target children's average age was 70.76 months $(S D=6.81)$. The percentage of boys and girls in the sample was nearly equal: 116 males and 118 females. The majority of mothers $(98.7 \%)$ reported being married. A total of 90 mothers reported being employed and 144 were not employed. More than half of the participants $(54.7 \%)$ reported an average annual income between KRW $24,000,000$ and KRW 50,000,000, slightly below the average household income of the general population in South Korea (Korean Statistical Information Service, 2015b). In this sample, the percentage of mothers who had obtained a college education (23\%) was lower than that of the general population in the Incheon area (40\%). 


\section{Procedures}

We obtained permission from the principals of four preprimary schools in the Incheon area to recruit parents for the study. Teachers in each classroom of the participating schools distributed an informational letter and a maternal survey to their students' homes. Mothers were informed that their participation was voluntary and that the study was solely for academic purposes. Participants completed the anonymous survey at home and mailed it directly to the research team. No identifying information was collected. Assessments of child social and academic competence were completed by the child's regular classroom teacher. All procedures were approved by the university's Institutional Review Board and all participating parents provided informed consent.

\section{Measures}

The survey consisted of several scales originally developed in English. A bilingual member of the American research team translated all survey items into Korean. A native Korean speaker who was fluent in English but not familiar with the purpose of the present study performed a backtranslation to refine item wording (Foster \& Martinez, 1995).

\section{Household income}

An estimate of household income was obtained using a 7-level scale $(1=$ Under KRW $12,000,000 ; 2=\mathrm{KRW}$ 12,010,000-24,000,000; $3=$ KRW 24,010,000-36,000,000; $4=\mathrm{KRW} 36,010,000-50,000,000 ; 5=\mathrm{KRW} 50,010,000$ $75,000,000 ; 6=$ KRW 75,010,000-100,000,000; $7=$ Over KRW 100,000,000).

\section{Mother education}

Mothers' educational attainment was assessed using a scale from 1 (no formal education) to 6 (graduate school or professional degree). We created a binary variable based on whether or not the respondent reported having a college degree.

\section{Mother labor force participation}

Mothers were asked to indicate the number of hours they engaged in the labor force per week. We used the raw score in our analyses.

\section{PSE}

Mothers' PSE was measured using the preprimary form of the Berkeley Parenting Self Efficacy Scale (BPSE-R) (Holloway et al., 2019). The Korean language version of the
BPSE-R demonstrated strong evidence of convergent and construct validity (S. Kim \& Holloway, 2018). Mothers used a 6-point Likert scale, ranging from 1 (not at all confident) to 6 (very confident) to indicate their PSE on the 7-item Parental Strategies subscale (e.g., "How confident are you that you can explain things so that your child will understand?") and on the 11-item Child Outcomes subscale (e.g., "How confident are you that you can teach your child to be polite?"). A PSE composite constructed by computing the mean of all 18 items demonstrated excellent internal consistency $($ Cronbach's alpha $=0.95)$.

\section{Mother life stress}

We used the 10-item version of the Perceived Stress Scale (Cohen \& Williamson, 1988, Cohen et al., 1983) in which respondents used a 5-point Likert scale from 1 (never) to 5 (very often) to indicate how often they had experienced thoughts and emotions related to environmental stressors during the previous month (e.g., "You could not cope with all the things that you had to do."). The PSS has demonstrated good internal consistency, a replicable factor structure, and evidence of construct validity in the United States (Cohen \& Janicki-Deverts, 2012) as well as in several European nations, Mexico, and the Russian Federation (e.g., Siqueira Reis et al., 2010). A Korean version of the PSS demonstrated good internal consistency (Cronbach's alpha $=0.82$ ) and test-retest reliability $(r=0.66)$, as well as a factor structure similar to that attained in studies of English speakers (E.-H. Lee, 2012). The PSS composite was calculated by computing the mean across all 10 items, except for one that was less strongly associated with the others ("able to control irritations in your life"). This scale exhibited good internal consistency (Cronbach's alpha $=0.85$ ).

\section{Mother marital satisfaction}

Marital satisfaction was assessed with the 10-item Marital Quality subscale of the ENRICH (Evaluation and Nurturing Relationship Issues, Communication and Happiness) Marital Satisfaction Scale (Fowers \& Olson, 1993). Each item of the Marital Quality subscale reflects one area of the marital relationship, including partner's personality, communication, conflict resolution, financial management, leisure activities, sexual relationship, children and parenting, family and friends, egalitarian roles, and religious orientation. To limit the length of our survey, we did not include the Idealistic Distortion subscale, which assesses respondents' tendency to idealize their relationship (e.g., "Our relationship is a perfect success".). Respondents rated their satisfaction on each item using a 5-point Likert scale ranging from 1 (strongly disagree) to 5 (strongly agree). The authors reported good test-retest reliability $(r=0.86)$ and 
Table 2 Correlations among study variables

\begin{tabular}{|c|c|c|c|c|c|c|c|c|c|}
\hline Variable & 1 & 2 & 3 & 4 & 5 & 6 & 7 & 8 & 9 \\
\hline Household income & - & & & & & & & & \\
\hline Child age (months) & -0.03 & - & & & & & & & \\
\hline Child gender ${ }^{\mathrm{a}}$ & -0.02 & 0.03 & - & & & & & & \\
\hline Mother age (years) & 0.06 & 0.08 & -0.06 & - & & & & & \\
\hline $\begin{array}{l}\text { Mother labor force } \\
\text { participation }\end{array}$ & $0.24^{* * * *}$ & 0.13 & 0.0 & -0.05 & - & & & & \\
\hline Mother education $^{\mathrm{b}}$ & $0.14^{*}$ & 0.0 & -0.04 & $0.20^{* *}$ & 0.11 & - & & & \\
\hline Mother life stress & -0.05 & -0.10 & 0.11 & -0.09 & -0.01 & -0.02 & - & & \\
\hline Mother marital satisfaction & 0.05 & 0.07 & -0.03 & 0.04 & -0.08 & 0.09 & $-0.60^{* * *}$ & - & \\
\hline Child competence & -0.02 & 0.06 & $0.36^{* * *}$ & 0.04 & 0.0 & 0.05 & 0.05 & 0.05 & - \\
\hline Mother PSE & -0.03 & 0.11 & 0.11 & 0.10 & 0.09 & -0.02 & $-0.26^{* * *}$ & $0.16^{*}$ & 0.13 \\
\hline
\end{tabular}

Note. Sample size varies slightly across variables (see Table 1)

${ }^{\mathrm{a}} 0=$ male; $1=$ female. ${ }^{\mathrm{b}} 0=$ below college degree attainment; $1=$ college degree attainment or above

${ }^{*} p<0.05 .{ }^{* *} p<0.01 .{ }^{* * *} p<0.001$ strong concurrent and construct validity, as indicated by significant associations between the ENRICH Marital Satisfaction Scale and other marital satisfaction measures (Fowers \& Olson, 1993). In a study conducted with Japanese mothers of young children, the Marital Quality subscale score was significantly correlated with PSE and perceived stress (Suzuki, 2010). In the present study, respondents were instructed to rate their satisfaction with a spouse or a partner with whom they were in a stable relationship. A total score was calculated as the mean of responses on all 10 items and demonstrated a high degree of internal consistency (Cronbach's alpha $=0.87$ ).

\section{Child competence}

We used all 25 items from the Social Competence ScaleTeacher Version (SCS; Conduct Problems Prevention Research Group, 1999) to assess children's prosocial skills (e.g., "resolves peer problems on own"), emotional selfregulation (e.g., "can calm down when excited or all wound up"), and cognitive/academic competence (e.g., "stays on task"). Teachers rated how well each item described the child on a 5-point Likert scale, with responses ranging from 1 (not at all) to 5 (very well). Validity information was provided in a study finding a significant association between SCS score and children's inattention and social skill deficits (Farmer et al., 2002). In our study, the child competence composite score was calculated by computing the mean across all 25 items $($ Cronbach's alpha $=0.97)$.

\section{Results}

All analyses were conducted using R Version 3.6.1 and RStudio Version 1.2.5001. We began by computing descriptive statistics and correlations among the study variables. To evaluate the predictors of PSE, we conducted three multiple regression analyses. Each analysis included sociodemographic control variables (household income, child age, child gender, maternal age, maternal labor force participation, and maternal education), as well as one of the following composites: life stress, marital satisfaction, or child competence. To evaluate the moderating effect of stress on the association of PSE to marital satisfaction and child competence, we conducted two additional multiple regressions. The first interaction model featured the sociodemographic variables, life stress, marital satisfaction, child competence, and the interaction between life stress and marital satisfaction. The second interaction model included the sociodemographic variables, life stress, marital satisfaction, child competence, and the interaction between life stress and child competence.

\section{Descriptive Statistics and Relations among Study Variables}

Descriptive statistics are presented in Table 1 and zeroorder correlations are presented in Table 2. None of the demographic variables were significantly associated with PSE. Attainment of a college degree was positively associated with maternal age $(r=0.20, p<0.01)$ and household income $(r=0.14, p<0.05)$. Women who worked more hours outside the home reported a higher household income $(r=0.24, p<0.001)$. Mothers reporting higher stress indicated less marital satisfaction $(r=-0.60, p<$ $0.001)$ and lower PSE $(r=-0.26, p<0.001)$. Mothers reporting higher marital satisfaction indicated higher PSE $(r=0.16, p<0.05)$. PSE was not significantly correlated to child competence. 


\section{Regression Analyses}

All variables were standardized before entering them into the regression equation (Aiken \& West, 1991). We explored the predictors of PSE in three analytic steps. First, we conducted a simultaneous multiple regression (Model 1) with the following predictors: household income, child age, child gender, maternal age, maternal weekly work hours, maternal education, life stress, marital satisfaction, and child competence. We then conducted two additional regressions, each of which included the same predictors as Model 1, in addition to the interaction of life stress with marital satisfaction in one (Model 2) and with child competence in the other (Model 3). Regression results from Model 1 and Model 2 are presented in Table 3.

\section{Research Questions 1 and 2: Association of marital satisfaction and child competence to maternal PSE}

Regression results from Model 1 show that neither marital satisfaction nor child competence was significantly related to PSE, controlling for all other variables in the model.

\section{Research Question 3: Association between general life stress and maternal PSE}

In Model 1, which controlled for all demographic variables as well as marital satisfaction and child competence, mothers experiencing greater life stress indicated having lower PSE $(\beta=-0.27, t(185)=-3.21, p<0.01)$. This model explained a significant proportion of the variance in PSE (adjusted $R^{2}=0.09, F(9,185)=3.12, p<0.01$ ).

\section{Research Question 4: Moderating association of life stress on relation of marital satisfaction and child competence to maternal PSE}

This question was examined by conducting two regression analyses, each containing all the variables included in Model 1 along with one of the following interaction terms: life stress X marital satisfaction (Model 2) and life stress X child competence (Model 3). As seen in Table 3, life stress moderated the association of marital satisfaction to PSE. In Model 2, the association of life stress to PSE was significant $\beta=-0.25, t(184)=-3.09, p<0.01$. The interaction between life stress and marital satisfaction was also significant $\beta=-0.16, t(184)=-2.88, p<0.01$. This model explained a significant proportion of the variance in PSE (Adjusted $R^{2}=0.12, F(10,185)=3.75, p<0.001$ ). An analysis of variance test comparing Model 1 and Model 2 confirmed that Model 2, with the addition of the interaction effect between life stress and marital satisfaction, demonstrated improved model fit $(F(1)=8.32, p=0.004)$. Model
Table 3 Regression results with mothers' PSE as outcome variable

\begin{tabular}{|c|c|c|}
\hline Effect & $\begin{array}{l}\text { Model } 1 \\
\text { Standardized } \\
\text { estimate }(\beta)\end{array}$ & $\begin{array}{l}\text { Model } 2 \\
\text { Standardized } \\
\text { estimate }(\beta)\end{array}$ \\
\hline Intercept & -0.01 & -0.10 \\
\hline \multicolumn{3}{|l|}{$\begin{array}{l}\text { Sociodemographic } \\
\text { characteristics }\end{array}$} \\
\hline Household income & -0.03 & -0.03 \\
\hline Child age (months) & 0.08 & 0.08 \\
\hline Child gender ${ }^{\mathrm{a}}$ & 0.06 & 0.05 \\
\hline Mother age (years) & 0.05 & 0.08 \\
\hline $\begin{array}{l}\text { Mother labor force } \\
\text { participation }\end{array}$ & 0.10 & 0.08 \\
\hline Mother education $^{\mathrm{b}}$ & -0.05 & -0.07 \\
\hline \multicolumn{3}{|l|}{ Family context } \\
\hline Mother life stress & $-0.27^{* *}$ & $-0.25^{* *}$ \\
\hline $\begin{array}{l}\text { Mother marital } \\
\text { satisfaction }\end{array}$ & 0.04 & 0.05 \\
\hline $\begin{array}{l}\text { Life stress*marital } \\
\text { satisfaction }\end{array}$ & - & $-0.16^{* *}$ \\
\hline \multicolumn{3}{|l|}{ Child characteristics } \\
\hline Child competence & 0.14 & 0.14 \\
\hline
\end{tabular}

3 , examining the interaction of life stress by child competence, was not significantly associated with PSE.

To better understand these findings, we conducted additional analyses examining the characteristics of women experiencing differing levels of life stress. To do so, we divided the respondents into three groups based on their rating of life stress (Fig. 1). The life stress of respondents in the low-stress group fell one standard deviation below the mean level of stress $(n=31)$. Respondents included in the medium-stress group had stress ratings that fell within one standard deviation of the mean stress level $(n=169)$ and those in the high-stress group reported stress ratings one standard deviation above the mean stress level $(n=31)$.

A series of one-way ANOVA tests indicated no differences among the three stress groups in terms of any sociodemographic characteristic (household income, child age, child gender, maternal age, maternal labor force participation, and maternal education), nor did they differ in terms of their child's competence. In line with the findings of the regression analysis examining the association of PSE to the interaction of life stress and marital satisfaction (Model 2), mothers across the three stress groups did differ with respect to their marital satisfaction. Mothers in the low-stress group reported the highest marital satisfaction $(M=4.17)$, followed by those in the medium-stress group $(M=3.53)$, then 
Fig. 1 Simple slopes and 95\% confidence intervals by stress group

Fig. 2 Marital relationship satisfaction by stress group
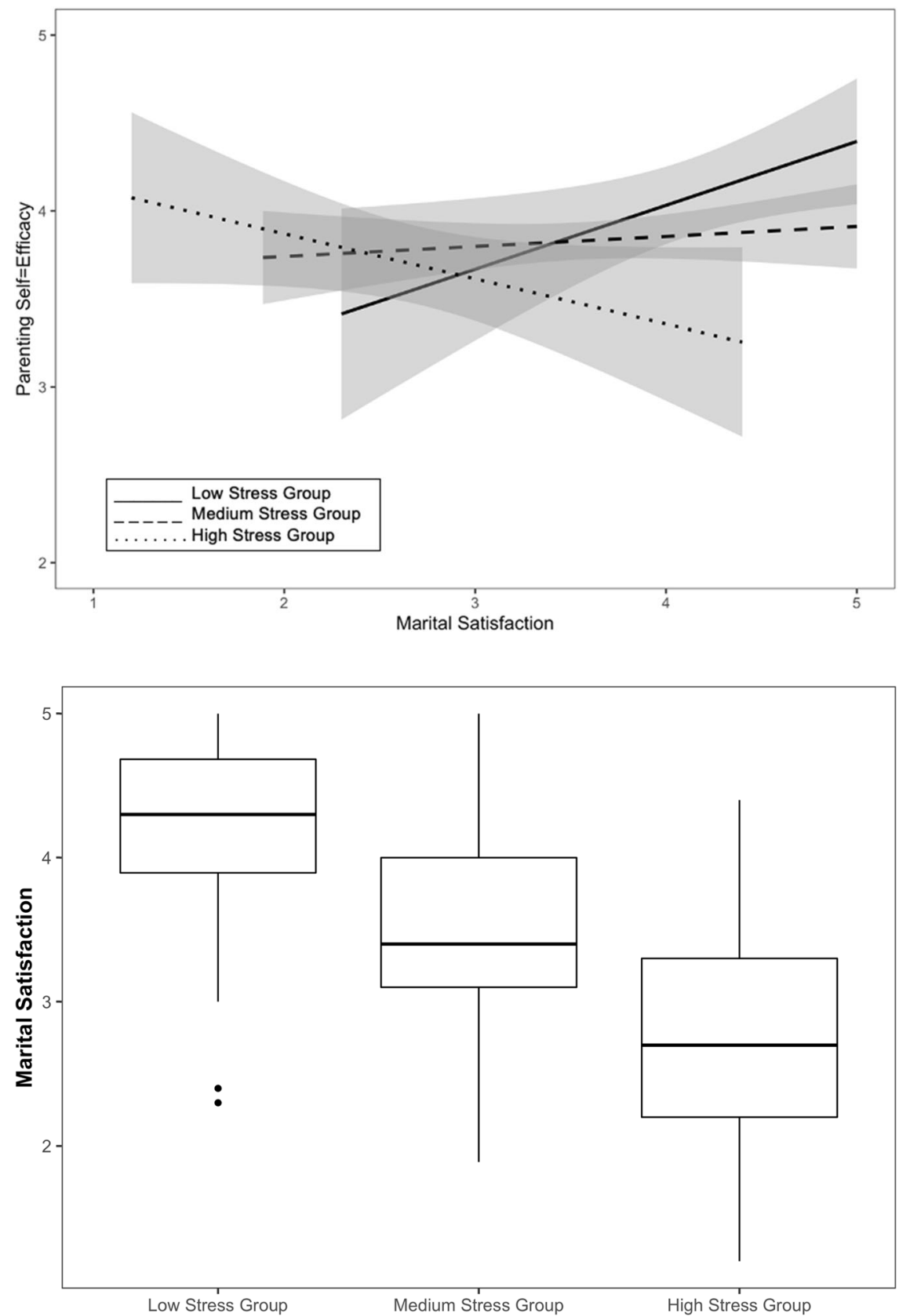

by those in the high-stress group $(M=2.71)$ (see Fig. 2). A Tukey Honest Significant Difference post-hoc test (Tukey's HSD) yielded significant variation on marital satisfaction mean scores across the three stress groups (medium vs. low $=-0.64$; high vs. low $=-1.46$; high vs. medium $=-0.82$; all $p s<0.001)$.

Standardized regression analyses with a single predictor (marital satisfaction) were run for the low-, medium-, and high-stress groups to test the moderation effect of stress on the relationship between marital satisfaction and PSE and to derive equations for the simple slopes displayed in Fig. 1. Mothers in the low-stress group showed a positive association between marital satisfaction and PSE $(\beta=0.49$, $t(29)=2.40, p=0.02)$. Conversely, in the high-stress group, mothers who were more satisfied with the marital relationship reported lower PSE $(\beta=-0.64, t(29)=-2.70$, $p=0.01)$. In the medium-stress group, mothers' marital satisfaction was not significantly related to PSE (i.e., the slope of marital satisfaction regressed on PSE was not significantly different from zero). Differences between the simple slopes were calculated by dividing a $t$-value for the difference between slopes by the pooled standard error (Robinson et al., 2013). All simple slopes were significantly different from each other: low vs. medium $(t(198)=2.85$, 
$p=0.002)$; low vs. high $(t(60)=7.40, p<0.001)$; medium vs. high stress $(t(198)=8.22, p<0.001)$.

To confirm the robustness of this moderation effect, we performed a second comparison, this time dividing mothers into three stress groups with an equal number of mothers in each group (rather than using one standard deviation above and below the mean to define the group cut points). Like the analysis described above, three separate regression analyses with a single predictor were performed using this new categorization of stress groups, with findings replicating those found in the previous analysis. Mothers in the lowstress group who reported higher marital satisfaction also indicated higher PSE $(\beta=0.40, t(75)=2.26, p=0.03)$. For mothers in the high-stress group, higher marital satisfaction was associated with lower PSE $(\beta=-0.33, t(74)=-2.14$, $p=0.04$ ). For mothers in the medium-stress group, there was not a significant relationship between marital satisfaction and PSE.

\section{Discussion}

This study helps address a gap in our understanding of how PSE is constructed within a parent's sociocultural context as well as within a given family context. We explored the correlates of PSE in a sample of mothers from South Korea, a country experiencing profound sociocultural changes and pressures on family life. We focused specifically on mothers' experience of life stress and the extent to which it is associated with PSE. Additionally, we examined whether mothers who were more satisfied with their marital relationship and those with higher teacher-rated child competence also experienced higher PSE. Finally, we sought to understand whether the relationship of PSE to marital satisfaction and to children's social and academic competence was moderated by mothers' experience of life stress.

Overall, our correlation results revealed that mothers experiencing more stressful lives also reported lower PSE and were far less satisfied with their marital relationship than less stressed women. The relationship between life stress and PSE remained significant even after we controlled for six important sociodemographic characteristics, as well as marital satisfaction and child competence. Our finding that mothers who experienced greater life stress also rated themselves as less efficacious parents is consistent with findings from Erdwins and colleagues (2001). The association that we documented between stress and PSE as well as the significant (but weak) association between marital satisfaction and PSE support the basic tenets of efficacy theory (Bandura, 1997). Our findings concerning marital satisfaction are also consistent with other studies from the United States and Japan, which found that PSE is associated with receiving instrumental and emotional support from a spouse or intimate partner (e.g., Merrifield \& Gamble, 2012, Suzuki et al., 2009).

However, in multivariate models that included family characteristics as well as life stress, the association between PSE and marital satisfaction did not remain significant. This finding mirror results from a study of Chinese parents showing a significant bivariate association between PSE and marital satisfaction that did not hold up in a multivariate model (Kwok et al., 2015). Collinearity was certainly a concern in our study given the high correlation between the independent variables of life stress and marital satisfaction. The findings may also have been stronger if we had used domain-specific measures of stress rather than a measure assessing life stress. For instance, if a mother's stress is directly related to, say, a difficult relationship at work, then the effect of husband support on PSE may be weak compared to a situation in which stress is more closely related to childrearing.

The results did not support our hypothesis that child competence is related to PSE. This is surprising, given that previous studies conducted in South Korea and the United States found that parents whose children had more behavioral problems reported lower PSE (e.g., H.M. Kim \& Doh, 2004, Shim \& Lim, 2019, Troutman et al., 2012). The construct of child competence is complex, and findings may differ depending on how it is operationalized and which specific predictors are tested in the research. In our study, teachers provided an assessment of child competence that included not only social and behavioral competence but also academic skills. Additionally, we may have found an indirect relation between child competence and PSE if we had also assessed parenting style and behaviors. For example, in their study of South Korean fathers, Shim and Lim (2019) found that PSE was related to warm and firm parenting. Warm parenting was in turn related to children's externalizing and internalizing behavior. These authors found no direct relationship between fathers' PSE and children's behavioral problems. Similarly, in their study of South Korean mothers and kindergartners, H.M. Kim and Doh (2004) found that PSE was related to children's social competence indirectly through parenting behavior.

Another factor that may have affected our findings is that our respondents likely had seen relatively little evidence concerning their young children's behavior with peers in the school setting, or of their academic skills. Our participants may have viewed their children's academic and social competence differently than did their teachers, making it less likely that teacher assessment would be related to any evaluation mothers might make of their own parenting (Leerkes \& Burney, 2007). It would be interesting to explore these relationships in families with slightly older children when public evaluation of children's achievement is a common practice and hence available to children's own parents. 
We also examined the moderating effect of life stress on the relation of PSE to marital satisfaction and to children's social and academic competence. We found that it was the interaction of life stress and marital satisfaction that bore a relationship to PSE. Specifically, for mothers reporting low levels of stress, higher marital satisfaction was positively related to higher PSE. However, it was surprising that we found no such relationship for women in the medium-stress group and that among the most highly stressed women, lower marital satisfaction was related to higher PSE. From post-hoc analyses examining differences in marital satisfaction between mothers in the three stress groups, we know that these women in the highly stressed group reported the lowest marital satisfaction compared to those in the lowand medium-stress groups.

It is possible that mothers reporting high stress may opt to meet the demands of either their maternal or their spousal role if they feel that they do not have the physical or psychological capacity to enact both roles fully. In this sense, mothers experiencing very little marital satisfaction may focus exclusively on childrearing as a way of coping during stressful conditions, and thus experience a relatively strong sense of PSE (A.-N. Kim \& Kwon, 2014). This coping strategy may be particularly available to South Korean women, who have traditionally received little support from their husbands in terms of childrearing and whose role as a mother may be viewed as primary over their marital satisfaction.

This finding suggests that South Korean mothers experiencing high levels of stress may be best supported by interventions that are tailored to their individual needs, rather than a one-size-fits-all approach to family intervention. Additionally, family practitioners and policymakers should be mindful that family support programs need to take a holistic approach to support highly stressed mothers, such that interventions aid spouses in balancing their roles are parents and partners, for example by building positive conflict resolution skills, healthy relationship skills, and developing more open communication.

The current study had several limitations. Our study depended on instruments developed by investigators working in the United States. When possible, it is advisable for researchers to conduct formal validation studies to ensure that measures developed elsewhere are appropriate for the focal societal context or to develop culturally specific measures. A second limitation was that we did not use all subscales of the ENRICH scale, given that we were mindful of burdening busy parents with a lengthy survey. Additionally, our findings regarding marital satisfaction may have been clearer if we had used a measure that focused more extensively on satisfaction with parenting support. If our sample had been larger, we would have had more statistical power to contrast the characteristics and behaviors of mothers in our low, medium, and high-stress groups.

While our exploratory analyses are helpful in pinpointing factors related to South Korean mothers' PSE, the cross-sectional nature of this study precludes conclusions about the causal role of marital satisfaction and life stress on PSE. Longitudinal studies or intervention research could be especially helpful in refining a causal model, although it is likely that these relationships have a strong transactional component. It should also be noted that we utilized a convenience sample that drew from one urban region. We anticipate that future studies will include participants from diverse geographic locations, family structures, and socioeconomic levels within South Korea. Future research might also consider other forms of child-related support, including that offered by extended family, neighbors, and friends, institutions, and communities (Hong \& Lee, 2019). In this vein, Suzuki et al. (2009) found that the PSE of Japanese mothers was higher among those who experienced greater support from close friends. In summary, future studies exploring the conditions that affect parenting should be based on an understanding of the relevant cultural context.

Funding This project was funded in part by a faculty research grant to Susan D. Holloway from the University of California, Berkeley.

\section{Compliance with Ethical Standards}

Conflict of Interest The authors declare no competing interests.

Consent to Participate Informed consent was obtained from all individuals included in the study.

Ethical Approval The questionnaire and methodology for this study were approved by the University of California Berkeley Committee for the Protection of Human Subjects Protocol ID 2011-08-3478. The procedures used in this study adhere to the tenets of the Declaration of Helsinki.

Publisher's note Springer Nature remains neutral with regard to jurisdictional claims in published maps and institutional affiliations.

Open Access This article is licensed under a Creative Commons Attribution 4.0 International License, which permits use, sharing, adaptation, distribution and reproduction in any medium or format, as long as you give appropriate credit to the original author(s) and the source, provide a link to the Creative Commons license, and indicate if changes were made. The images or other third party material in this article are included in the article's Creative Commons license, unless indicated otherwise in a credit line to the material. If material is not included in the article's Creative Commons license and your intended use is not permitted by statutory regulation or exceeds the permitted use, you will need to obtain permission directly from the copyright holder. To view a copy of this license, visit http://creativecommons. org/licenses/by/4.0/. 


\section{References}

Aiken, L. S., \& West, S. G. (1991). Multiple regression: Testing and interpreting interactions. Sage Publications, Inc.

Bak, S.-H. (2017, January 9). 83 percent of five-year-olds in Korea go to hagwon. The Korea Herald. http://www.koreaherald.com/ view.php?ud=20170109000747.

Bandura, A. (1977). Self-efficacy: Toward a unified theory of behavioral change. Psychological Review, 84, 191-215. https://doi. org/10.1037/0033-295X.84.2.191.

Bandura, A. (1995). Exercise of personal and collective efficacy in changing societies. In A. Bandura (Ed.), Self-efficacy in changing societies (pp. 1-45). Cambridge University Press. https://doi.org/ 10.1017/CBO9780511527692.003.

Bandura, A. (1997). Self-efficacy: The exercise of control. W.H. Freeman and Company.

Bornstein, M. H., Hendricks, C., Hahn, C. S., Haynes, O. M., Painter, K. M., \& Tamis-LeMonda, C. S. (2003). Contributors to selfperceived competence, satisfaction, investment, and role balance in maternal parenting: A multivariate ecological analysis. Parenting: Science and Practice, 3(4), 285-326. https://doi.org/10. 1207/s15327922par0304_2.

Bouchard, G., Lee, C. M., Asgary, V., \& Pelletier, L. (2007). Fathers' motivation for involvement with their children: A selfdetermination perspective. Fathering, 5, 25-41. https://doi.org/ 10.3149/fth.0501.25.

Bronfenbrenner, U., \& Morris, P. A. (2006). The bioecological model of human development. In W. Damon \& R. M. Lerner (Eds.), Handbook of child psychology: Theoretical models of human development (pp. 793-828). John Wiley \& Sons Inc.

Cha, S.-E., \& Song, Y.-J. (2017). Time or money: The relationship between educational attainment, income contribution, and time with children Among Korean fathers. Social Indicators Research: An International and Interdisciplinary Journal for Quality-of-Life Measurement, 134(1), 195-218. https://doi.org/10.1007/s11205016-1414-2.

Chau, V., \& Giallo, R. (2015). The relationship between parental fatigue, parenting self-efficacy and behaviour: Implications for supporting parents in the early parenting period. Child: Care, Health and Development, 41(4), 626-633. https://doi.org/10. 1111/cch.12205.

Choi, S.-W., Yellow Horse, A. J., \& Yang, T.-C. (2018). Family policies and working women's fertility intentions in South Korea. Asian Population Studies, 14(3), 251-270. https://doi.org/10. 1080/17441730.2018.1512207.

Chung, G. H., Lee, H., Lee, J., \& Lee, K. (2015). A mediational model of school involvement, knowledge about a child's school life, and parental efficacy among South Korean mothers. Journal of Child and Family Studies, 24(4), 899-908. https://doi.org/10.1007/ s10826-014-9900-0.

Cohen, S., Gottlieb, B. H., \& Underwood, L. G. (2000). Social relationships and health. In S. Cohen, L. G. Underwood, \& B. H. Gottlieb (Eds.), Social support measurement and intervention: A guide for health and social scientists (pp. 3-25). Oxford University Press. https://doi.org/10.1093/med:psych/9780195126709.003.0001.

Cohen, S., \& Janicki-Deverts, D. (2012). Who's stressed? Distributions of psychological stress in the United States in probability samples from 1983, 2006, and 2009. Journal of Applied Social Psychology, 42(6), 1320-1334. https://doi.org/10.1111/j.15591816.2012.00900.x.

Cohen, S., Kamarck, T., \& Mermelstein, R. (1983). A global measure of perceived stress. Journal of Health and Social Behavior, 385-396. https://doi.org/10.2307/2136404.

Cohen, S., \& Williamson, G. (1988). Perceived stress in a probability sample of the US. In Spacapam S., \& Oskamp, S., (Eds.), The social psychology of health: claremont symposium on applied social psychology (pp. 31-67). Sage.

Cohen, S., \& Wills, T. A. (1985). Stress, social support and the buffering hypothesis. Psychological Bulletin, 98, 310-357. https:// doi.org/10.1037/0033-2909.98.2.310.

Coleman, P. K., \& Karraker, K. H. (1998). Self-efficacy and parenting quality: Findings and future applications. Developmental Review, 18, 30-46. https://doi.org/10.1006/drev.1997.0448.

Coleman, P. K., \& Karraker, K. H. (2000). Parenting self-efficacy among mothers of school-age children: Conceptualization, measurement, and correlates. Family Relations, 49, 13-24. https://doi. org/10.1111/j.1741-3729.2000.00013.x.

Coleman, P. K., \& Karraker, K. H. (2003). Maternal self-efficacy beliefs, competence in parenting, and toddlers' behavior and developmental status. Infant Mental Health Journal, 24(2), 126-148. https://doi.org/10.1002/imhj.10048.

Conduct Problems Prevention Research Group. (1999). Initial impact of the fast track prevention trial for conduct problems: II. Classroom effects. Journal of Consulting and Clinical Psychology, 67 (5), 648-657. https://doi.org/10.1037/0022-006X.67.5.648.

Donovan, W. L., \& Leavitt, L. A. (1989). Maternal self-efficacy and infant attachment: Integrating physiology, perceptions, and behavior. Child Development, 60(2), 460-472. https://doi.org/10. 2307/1130990.

Dumka, L. E., Gonzales, N. A., Wheeler, L. A., \& Millsap, R. E. (2010). Parenting self-efficacy and parenting practices over time in Mexican American families. Journal of Family Psychology, 24 (5), 522-531. https://doi.org/10.1037/a0020833.

Erdwins, C. J., Buffardi, L. C., Casper, W. J., \& O’Brien, A. S. (2001). The relationship of women's role strain to social support, role satisfaction, and self-efficacy. Family Relations: An Interdisciplinary Journal of Applied Family Studies, 50(3), 230-238. https://doi.org/10.1111/j.1741-3729.2001.00230.x.

Erel, O., \& Burman, B. (1995). Interrelatedness of relationship relations and parent-child relations: A meta-analytic review. Psychological Bulletin, 118(1), 108-132. https://doi.org/10.1037/ 0033-2909.118.1.108.

Farmer, A. D., \& Bierman, K. L., Conduct Problems Prevention Research Group. (2002). Predictors and consequences of aggressive-withdrawn problem profiles in early grade school. Journal of Clinical Child \& Adolescent Psychology, 31(3), 299-311. https://doi.org/10.1207/S15374424JCCP3103_02.

Foster, S. L., \& Martinez, C. R. (1995). Ethnicity: Conceptual and methodological issues in child clinical research. Journal of Clinical Child Psychology, 24(2), 214-226. https://doi.org/10. 1207/s15374424jccp2402_9.

Fowers, B. J., \& Olson, D. H. (1993). ENRICH relationship satisfaction scale: a brief research and clinical tool. Journal of Family Psychology, 7(2), 176. https://doi.org/10.1037/0893-3200.7.2.176.

Gross, D., \& Tucker, S. (1994). Parenting confidence during toddlerhood: A comparison of mothers and fathers. The Nurse Practitioner, 19(10), 25-29. https://doi.org/10.1097/00006205199410000-00004.

Harkness, S., Moscardino, U., Blom, M. J., Huitrón, B., Johnston, C. A., Sutherland, M. A., Axia, G., \& Palacios, J. (2007). Cultural models and developmental agendas: implications for arousal and self-regulation in early infancy. Journal of Developmental Processes, 1(2), 5-39.

Holloway, S. D., Suzuki, S., Kim, S., Nagase, A., Wang, Q., Campbell, E. J., Golshirazi, M., Iwatate, K., \& Nishizaka, S. (2019). Development and cross-national validation of a revised version of the Berkeley Parenting Self-efficacy Scale. Early Childhood Research Quarterly, 47, 309-320. https://doi.org/10.1016/j. ecresq.2018.12.016

Hong, Y.-J., \& Lee, K. (2019). The effect of parenting stress on social interactive parenting with a focus on Korean employed mothers' 
parenting support from ecological contexts. Children and Youth Services Review, 96, 308 https://doi.org/10.1016/j.childyouth. 2018.10.038.

Jeong, Y., \& Jeon, G. (2018). Relationship between perfectionism and parenting behavior of working mothers: Double mediating effects of work-family conflict and parenting guilt. Family and Environment Research, 56(4), 377-390. https://doi.org/10.6115/fer.2018.027.

Jones, T. L., \& Prinz, R. J. (2005). Potential roles of parental self-efficacy in parent and child adjustment: A review. Clinical Psychology Review, 25(3), 341-363. https://doi.org/10.1016/j.cpr.2004.12.004.

Kim, A.-N., \& Kwon, Y.-S. (2014). Effect of employed mother's work-mother role conflict on parenting stress: Mediation effect of parental satisfaction. Journal of Digital Convergence, 12(10), 375-384. https://doi.org/10.14400/JDC.2014.12.10.375.

Kim, C. (2020). Lost Seoul: South Korean middle-class dreams spoiled by soaring house prices. Reuters. https://www.reuters. com/article/us-southkorea-economy-middleclass-insigh/lostseoul-south-korean-middle-class-dreams-spoiled-by-soaringhouse-prices-idUSKCN2520K8.

Kim, H. M., \& Doh, H.-S. (2004). Maternal parenting stress, efficacy, and behavior: Relations to children's social competence. Korean Journal of Child Studies, 25(6), 279-298.

Kim, S., \& Holloway, S. D. (2018). Parenting and young children's emotional self-regulation in urban Korean families. Journal of Early Childhood Research, 16, 305-318. https://doi.org/10.1177/ $1476718 X 18775759$.

Koo, H. (2007). The changing faces of inequality in South Korea in the age of globalization. Korean Studies, 1-18. https://doi.org/10. 1353/ks.2008.0018.

Korean Statistical Information Service. (2015a). Gender, age, and educational level of population. http://kosis.kr/statHtml/statHtml. do?orgId=101\&tblId=DT_1IN1004\&conn_path=I2 .

Korean Statistical Information Service. (2015b). Household income and expenditure survey. http://kosis.kr/eng/statisticsList/sta tisticsList_01List.jsp?vwcd=MT_ETITLE\&parentId= C\#SubCont.

Kwok, S. Y. C. L., Cheng, L., Chow, B. W. Y., \& Ling, C. C. Y. (2015). The spillover effect of parenting on relationship satisfaction among Chinese mothers. Journal of Child and Family Studies, 24(3), 772-783. https://doi.org/10.1007/s10826-013-9888-х.

Kwon, Y. I., \& Roy, K. M. (2007). Changing social expectations for work and family involvement among Korean fathers. Journal of Comparative Family Studies, 38(2), 285-305. https://doi.org/10. 3138/jcfs.38.2.285.

Lee, E.-H. (2012). Review of the psychometric evidence of the Perceived Stress Scale. Asian Nursing Research, 6(4), 121-127. https://doi.org/10.1016/j.anr.2012.08.004.

Lee, S., \& Shouse, R. C. (2011). The impact of prestige orientation on shadow education in South Korea. Sociology of Education, 84, 212-224. https://doi.org/10.1177/0038040711411278.

Leerkes, E. M., \& Burney, R. V. (2007). The development of parenting efficacy among new mothers and fathers. Infancy, 12(1), 45-67. https://doi.org/10.1111/j.1532-7078.2007.tb00233.x.

Merrifield, K. A., \& Gamble, W. C. (2012). Associations among marital qualities, supportive and undermining coparenting, and parenting self-efficacy: Testing spillover and stress-buffering processes. Journal of Family Issues, 34(4), 510-533. https://doi. org/10.1177/0192513X12445561.

National Center for Education Statistics. (2020). International Comparisons: Enrollment rates by country. https://nces.ed.gov/progra ms/coe/indicator_cgh.asp.

OECD Education GPS. (2021). Korea: Overview of the Education System. https://gpseducation.oecd.org/CountryProfile?primaryCo untry $=$ KOR\& treshold $=10 \&$ topic $=$ EO.

Park, H., Byun, S.-Y., \& Kim, K.-K. (2011). Parental involvement and students' cognitive outcomes in Korea: Focusing on private tutoring. Sociology of Education, 84(1), 3-22. https://doi.org/10. 1177/0038040710392719.

Robinson, C. D., Tomek, S., \& Schumacker, R. E. (2013). Tests of moderation effects: Difference in simple slopes versus the interaction term. Multiple Linear Regression Viewpoints, 39(1), $16-24$.

Schneewind, K. A. (1995). Impact of family processes on control beliefs. In A. Bandura (Ed.), Self-efficacy in changing societies (pp. 114-148). Cambridge University Press. https://doi.org/10. 1017/CBO9780511527692.003.

Shim, S. Y., \& Lim, S. A. (2019). Paternal self-efficacy, fathering, and children's behavioral problems in Korea. Journal of Child and Family Studies, 28(3), 851-859. https://doi.org/10.1007/s10826018-01310-7.

Shwalb, D. W., Nakazawa, J., Yamamoto, T., \& Hyun, J.-H. (2004). Fathering in Japanese, Chinese, and Korean cultures: A review of the research literature. In M. E. Lamb (Ed.), The role of the father in child development (4th ed., pp. 146-181). John Wiley \& Sons Inc.

Siqueira Reis, R., Ferreira Hino, A. A., \& Romélio Rodriguez Añez, C. (2010). Perceived stress scale: reliability and validity study in Brazil. Journal of Health Psychology, 15(1), 107-114. https://doi. org/10.1177/1359105309346343.

Suzuki, S. (2010). The effects of relationship support, social network support, and parenting stress on parenting: Self-efficacy among mothers of young children in Japan. Journal of Early Childhood Research, 8(1), 40-66. https://doi.org/10.1177/1476718X09345506.

Suzuki, S., Holloway, S. D., Yamamoto, Y., \& Mindnich, J. (2009). Parenting self-efficacy and social support in Japan and the United States. Journal of Family Issues, 30, 1505-1526. https://doi.org/ 10.1177/0192513X09336830.

Troutman, B., Moran, T. E., Arndt, S., Johnson, R. F., \& Chmielewski, M. (2012). Development of parenting self-efficacy in mothers of infants with high negative emotionality. Infant Mental Health Journal, 33(1). https://doi.org/10.1002/imhj.20332.

World Economic Forum. (2019). The Global Gender Gap Report 2020. https://reports.weforum.org /global-gender-gap-report2020.

Yeung, W.-J. (2013). Asian fatherhood. Journal of Family Issues, 34, 143-160. https://doi.org/10.1177/0192513X12461133.

Students' private education spending surges to 10-year-high in 2019. (2020, March 10). Yonhap News Agency. https://en.yna.co.kr/ view/AEN20200310006100315.

Yoo, J. (2020). Gender role attitude, communication quality, and marital satisfaction among Korean adults. Journal of Family Studies. https://doi.org/10.1080/13229400.2020.1791230. 